\title{
Investigação sorológica da influenza tipos A e B em estudantes universitários, Brasil ${ }^{*}$
}

\author{
Serological surveillance of influenza $A$ and $B$, at the university students, Brazil.
}

\author{
Dalva A. P. Mancini" ${ }^{\star \star}$, Valéria R. Tavares ${ }^{\star \star}$, Clarice P. M. Espindola**
}

\begin{abstract}
MANCINI, D.A.P. et al. Investigação sorológica da influenza tipos A e B em estudantes universitários, Brasil. Rev. Saúde públ., S. Paulo, 25: 468-72, 1991. Levantamento sorológico realizado em 200 estudantes da Universidade de São Paulo, nos anos de 1984 e 1985, demonstrou ampla prevalência sorológica do vírus da influenza tipos A e B. Os anticorpos dos indivíduos foram detectados pela técnica de Hemólise Radial Simples (HRS), cujas médias aritméticas de títulos foram maiores entre as cepas dos subtipos $\left(\mathrm{H}_{1} \mathrm{~N}_{1}\right)$ e $\left(\mathrm{H}_{3} \mathrm{~N}_{2}\right)$ do vírus da influenza tipo $\mathrm{A}$, mais recentemente isoladas da população. Porém, com relação ao tipo $\mathrm{B}$, deste vírus, a situação foi inversa, pois apesar da cepa B/Engl./ $847 / 73$ ser a mais antiga incidente, revelou melhor reatogenicidade sobre as demais cepas avaliadas e de acordo com a doutrina do "Pecado original antigênico", é suposto que tenha sido responsável pela primo infecção na maioria do grupo investigado. A avaliação sorológica dos subtipos do vírus influenza tipos A e B, desta população, revelou índices de anticorpos de baixos títulos HRS (2,5 a 3,5 mm) e de altos títulos $(\geq 4,0 \mathrm{~mm})$ que estão relacionadas ao menor e maior nível de proteção à infecção. Sendo que a capacidade individual da imunidade e da persistência de anticorpos contra o vírus, dependeram da atualidade e frequiência de exposição à influenza.
\end{abstract}

Descritores: Influenza, epidemiologia. Métodos soroepidemiológicos.

\section{Introdução}

No Brasil, como na maior parte do mundo, a influenza tem se apresentado de forma epidêmica $e$ endêmica, atingindo indivíduos de todas as idades ${ }^{2,6}$, 11, 25 . A ocorrência dos surtos de gripe tem acarretado problemas de extrema gravidade chegando até a casos letais, principalmente entre subnutridos e nos grupos de risco encontrados em creches, asilos e hospitais ${ }^{1,3,4,5,13,20}$.

Devido à circulação de um grande número de variantes antigênicas do vírus da influenza, conseqüentes da sua acentuada capacidade de recombinação genética, é que a maioria da população continua susceptível a alguma cepa deste vírus ${ }^{1,9}$. Além do que, a susceptibilidade mais acentuada em crianças e idosos à infecção viral, decorrente da diminuição acelerada dos anticorpos, faz com que estes grupos sejam mais atingidos pelas infecções gripais ${ }^{2,3,12}$.

\footnotetext{
* Subvencionado pelo Conselho Nacional de Desenvolvimento Científico e Tecnológico (CNPq). Processo $\mathrm{n}^{9} 1.171 .379 / 85$

** Seção de Riquétsias do Instituto Butantan - São Paulo, SP - Brasil.

Separatas/Reprints: D.A.P. Mancini - Av. Vital Brasil, 1500-05503 - São Paulo, SP - Brasil

Publicação financiada pela FAPESP. Processo Medicina 90/4602-1
}

A caracterização das cepas do vírus da influenza que mais atingem uma população, é de grande importância no processo de monitoramento da extensão epidemiológica tanto do ponto de vista da sensibilidade da população a uma determinada cepa como, também, para fornecer elementos à seleção da composição de imunógenos ${ }^{10,13,18,20,26,29}$.

Dentro destas proposições, foram analisados soros de estudantes da Universidade de São Paulo, para se obter o levantamento sorológico de diferentes cepas dos tipos A e B do vírus da influenza. A análise sorológica através do teste ca Hemólise Radial Simples, tem demonstrado grande sensibilidade nas investigações da influenza ${ }^{7}$ 19.21. 28, Jando condições de caracterizar individualmente diferentes subtipos sorológicos do vírus. Estas informações permitem selecionar as cepas apropriadas para composição da vacina inativada contra gripe trivalente que usualmente é preparada no Instituto Butantan.

A Organização Mundial de Sáude informa e recomenda as cepas que devem compor a vacina contra gripe, porém ressalta a importância da avaliação dos subtipos incidentes no próprio meio, para completar esta orientação ${ }^{18}$.

\section{Materiais e Métodos}

Soros

Soros de 200 estudantes da Universidade de São 
Paulo, de faixa etária entre 19 e 25 anos, foram obtidos por punção venosa, invativados a $56^{\circ} \mathrm{C}$ por 30 min e mantidos congelados a $-20^{\circ} \mathrm{C}$ até serem submetidos ao teste.

O período da coleta de amostras deu-se entre os anos de 1984 e 1985.

Virus

Cepas de vírus influenza:

${ }^{*} \mathrm{~A} / \mathrm{SP} / 1 / 78=\mathrm{A} / \mathrm{Brazil} / 11 / 78\left(\mathrm{H}_{1} \mathrm{~N}_{1}\right) ;{ }^{*} \mathrm{~A} / \mathrm{SP} / 1 /$ $80=\mathrm{A} / \mathrm{Bang} \mathrm{Kok} / 1 / 79 /\left(\mathrm{H}_{3} \mathrm{~N}_{2}\right) ; \mathrm{A} / \mathrm{SP} / 1 / 81=\mathrm{A} /$ England/1333/80 $\left(\mathrm{H}_{1} \mathrm{~N}_{1}\right) ; \mathrm{A} / \mathrm{SP} / \mathbf{1} / 82=\mathrm{A} /$ Arizona/ $2 / 80\left(\mathrm{H}_{3} \mathrm{~N}_{2}\right) ; \mathrm{B} / \mathrm{SP} / 1 / 83=\mathrm{B} /$ Singapore$/ 222 / 79 ;$ e $\mathrm{B} /$ England $/ 847 / 73$, fornecidas pelo Instituto Adolfo Lutz de São Paulo e mantidas através de passagens em ovos embrionados de galinha, foram empregadas na avaliação sorológica.

\section{Teste de Hemólise Simples (HRS)}

O teste de HRS empregado nas titulações dos anticorpos nos soros baseou-se na técnica padronizada por Schild ${ }^{23}$ com algumas modificações ${ }^{14,17,19}$. ${ }^{21}$. O teste fundamenta-se na lise das hemácias de galo, sensibilizados com vírus e mediada pelo complemento (soro fresco de cobaia) quando na presença de anticorpos específicos do vírus. A reação é considerada positiva quando os valores das médias dos diâmetros dos halos de hemólise forem iguais ou superiores a $2,5 \mathrm{~mm}$. No teste controle, o concentrado viral foi substituído por líquido alantóide puro, seguindo-se a mesma metodologia para verificar ausência de reação.

\section{Resultados}

As frequiências de anticorpos contra as cepas do vírus da influenza, A/SP/1/78 $\left(\mathrm{H}_{1} \mathrm{~N}_{1}\right), \mathrm{A} / \mathrm{SP} / 1 / 81$ $\left(\mathrm{H}_{1} \mathrm{~N}_{1}\right), \mathrm{A} / \mathrm{SP} / 1 / 80\left(\mathrm{H}_{3} \mathrm{~N}_{2}\right)$, B/England/847/73 e B/ $\mathrm{SP} / 1 / 83$, detectados pela técnica de HRS, em soros de 200 estudantes se encontram registradas nas figuras 1 a 6 . A comparação das médias dos títulos de anticorpos obtidos das diferentes cepas virais, está demonstrada na Figura 7.

\section{Discussão e Conclusões}

Nos gráficos apresentados nas Figuras 1 e 2, observa-se a resposta de anticorpos contra o subtipo $\left(\mathrm{H}_{1} \mathrm{~N}_{1}\right)$ do vírus influenza tipo $\mathrm{A}$, detectada pelo teste HRS, onde $43,5 \%$ da população avaliada apresenta títulos de anticorpos HRS de 2,5 a 3,5

\footnotetext{
* Cepas recomendadas pela OMS, para composição da vacina contra gripe - $1985-1986^{27}$.
}

$\mathrm{mm}$, e $56,5 \%$ apresenta títulos de $\geq 4,0 \mathrm{~mm}$, para o subtipo $\left(\mathrm{H}_{1} \mathrm{~N}_{1}\right)$ isolado no ano de 1978.

Ao que se refere ao subtipo do ano de 1981, verifica-se $37,5 \%$ de indivíduos com títulos de anticorpos HRS de 2,5 a $3,5 \mathrm{~mm}$ e $62,5 \%$ com títulos de anticorpos HRS $\geq 4,0 \mathrm{~mm}$.

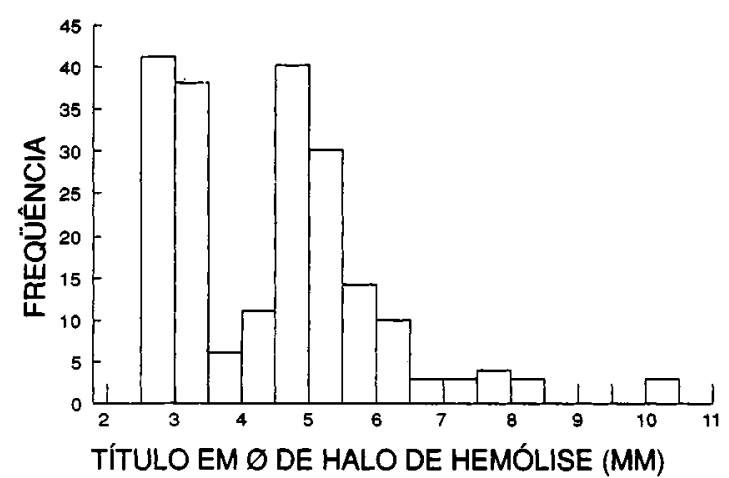

Flgura 1. Freqüência de anticorpos para vírus influenza A/SP/1/78 $\left(\mathrm{H}_{9} \mathrm{~N}_{1}\right)$ na população de estudantes universitários.

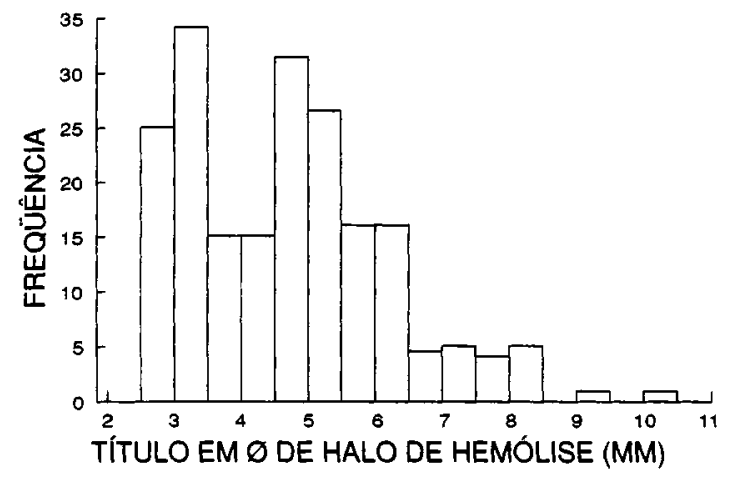

Figura 2. Freqüência de anticorpos para vírus influenza A/SP/2/81 $\left(\mathrm{H}_{1} \mathrm{~N}_{1}\right)$ na população de estudantes universitários.

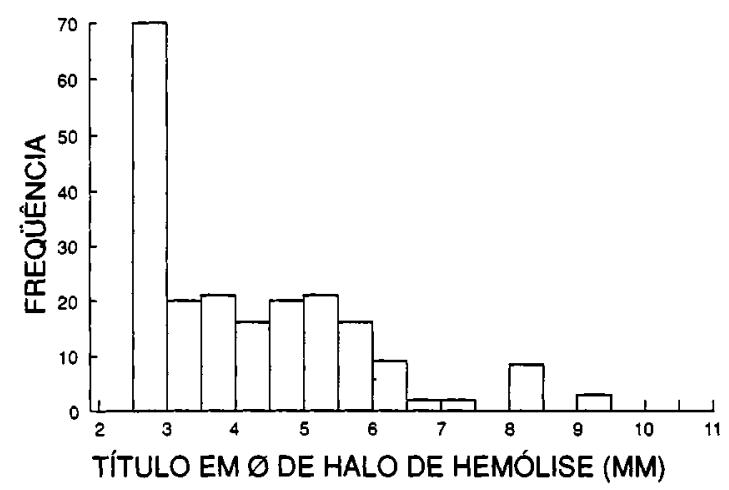

Figura 3. Freqüência de anticorpos para vírus influenza A/SP/1/80 $\left(\mathrm{H}_{3} \mathrm{~N}_{2}\right)$ na população de estudantes universitários. 


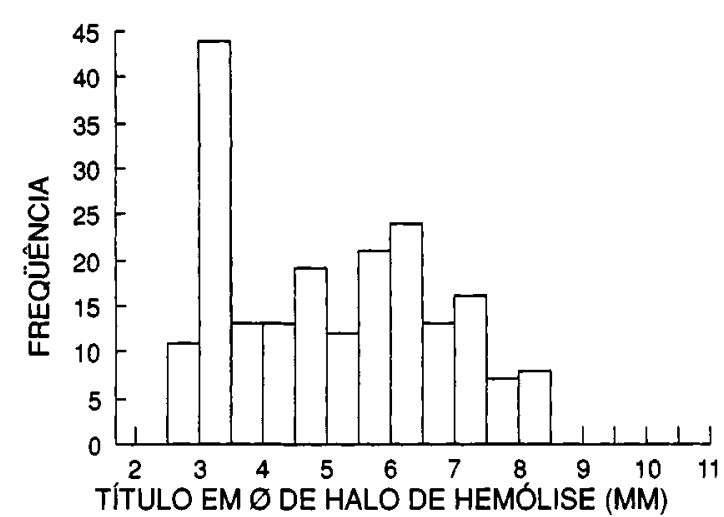

Figura 4. Freqüência de anticorpos para vírus influenza A/SP/1/82 $\left(\mathrm{H}_{3} \mathrm{~N}_{2}\right)$ na população de estudantes universitários.

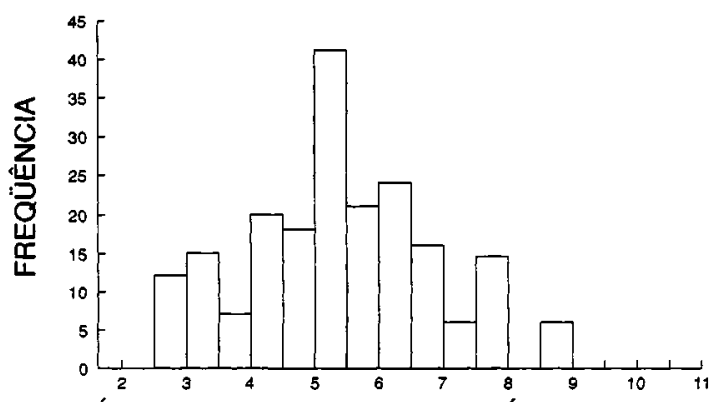

TÍTULO EM $\varnothing$ DE HALO DE HEMÓLISE (MM)

Figura 5. Freqüência de anticorpos para vírus influenza B/Engl/847/73 na população de estudantes universitários.

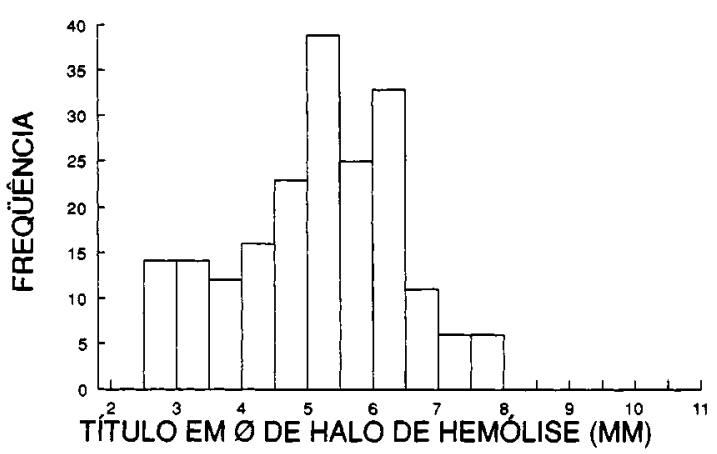

Figura 6. Freqüência de anticorpos para vírus influenza $\mathrm{B} / \mathrm{SP} / 1 / 83$ na população de estudantes universitários.

Nas Figuras 3 e 4 , onde se apresentam os gráficos que registram títulos de anticorpos obtidos pelo teste HRS, contra o subtipo $\left(\mathrm{H}_{3} \mathrm{~N}_{2}\right)$ do vírus influenza tipo $\mathrm{A}$, verifica-se que $55,5 \%$ dos indivíduos avaliados apresentam títulos de anticorpos HRS de 2,5 a $3,5 \mathrm{~mm}$ e $44,5 \%$ apresentam títulos de anticorpos $\mathrm{HRS} \geq 4,0 \mathrm{~mm}$, ao subtipo $\left(\mathrm{H}_{3} \mathrm{~N}_{2}\right)$ isolado em 1980. Com relação ao subtipo $\left(\mathrm{H}_{3} \mathrm{~N}_{2}\right)$ do

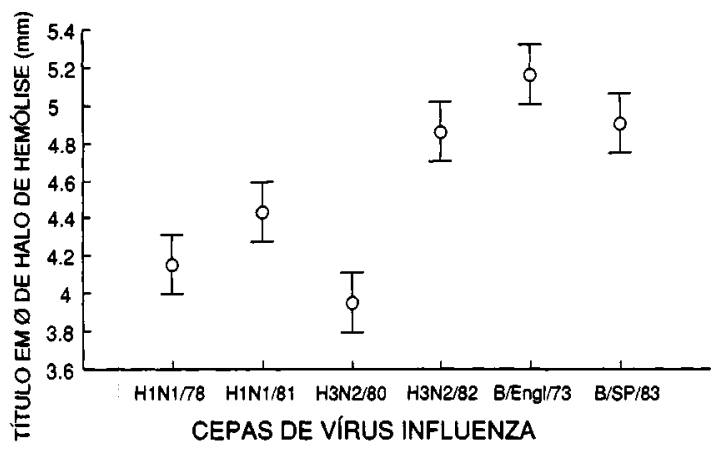

Figura 7. Títulos de anticorpos, pela Hemólise Radial Simples, de diferentes cepas do vírus influenza na populaçắo de estudantes universitários.

ano de 1982, verifica-se títulos de anticorpos HRS $\geq 4,0 \mathrm{~mm}$ em $65,5 \%$ dos indivíduos avaliados.

A resposta de anticorpos aos subtipos do vírus influenza tipo $B$ está representada nos gráficos das Figuras 5 e 6 , onde se observa que ao subtipo do ano de 1973 , obteve-se $17,5 \%$ de indivíduos com títulos de anticorpos HRS de 2,5 a $3,5 \mathrm{~mm}$ e $82,5 \%$ com títulos de anticorpos $\mathrm{HRS} \geq 4,0 \mathrm{~mm}$. Ao subtipa B do ano de 1983, verifica-se títulos de anticorpos HRS de 2,5 a $3,5 \mathrm{~mm}$, em $20,5 \%$ dos indivíduos analisados e 79,5\% destes com títulos de anticorpos $\mathrm{HRS} \geq 4,0 \mathrm{~mm}$.

Na Figura 7 estão demonstradas as médias dos títulos de anticorpos HRS contra os subtipos $\left(\mathrm{H}_{1} \mathrm{~N}_{1}\right)$ de 1978 e 1981 , subtipos $\left(\mathrm{H}_{3} \mathrm{~N}_{2}\right)$ de 1980 e 1982 do vírus influenza tipo A, e o tipo B dos anos de 1973 e 1983, onde se observa que o último subtipo A e os 2 subtipos B apresentam maiores médias de títulos de anticorpos contra o vírus influenza.

Os resultados obtidos durante a investigação sorológica, pelo teste de HRS, foram comparados aos resultados da placa controle, onde não se verificou a formação de halo de hemólise.

Observando-se os resultados apresentados nas Figuras de 1 a 6, verifica-se dois níveis de resposta de anticorpos contra o vírus influenza, um deles com títulos mais baixos (2,5 a $3,5 \mathrm{~mm})$ e outro com títulos mais altos ( $\geq 4,0 \mathrm{~mm}$ ), isto sugere que além da influência da capacidade imunogênica que cada cepa viral possui na indução de anticorpos, há também a variabilidade na persistência do nível de anticorpos de indivíduo para indivíduo ${ }^{14}$. Cabendo, ainda, relatar que o nível de anticorpos é suposto estar proporcionalmente relacionado ao grau de proteção à infecção por vírus influenza, como temos observado em monitoramento sorológico, após imunização contra gripe ${ }^{15}$. Portanto, apesar das diferenças de níveis de anticorpos detectadas na população investigada, fica evidente que todas as cepas do vírus influenza a atingiu em sua totalidade. 
Através da análise estatística dos resultados das titulações dos 200 soros de indivíduos sem histórico de vacinação contra influenza, apresentada na Figura 7, verificam-se médias aritméticas que apresentaram os seguintes valores: títulos de anticorpos de $4,14(\delta 1,43)$ para a cepa $\left(\mathrm{H}_{1} \mathrm{~N}_{1}\right) / 78$ e $4,42(\delta 1,49)$ para $\left(\mathrm{H}_{1} \mathrm{~N}_{1}\right) / 81$. Com relação às cepas $\left(\mathrm{H}_{3} \mathrm{~N}_{2}\right)$ as médias foram de $3,94(\delta 1,56)$ para $\left(\mathrm{H}_{3} \mathrm{~N}_{2}\right) / 80$ e 4,86 $(\delta 1,62)$ para $\left(\mathrm{H}_{3} \mathrm{~N}_{2}\right) / 82$. Quanto às cepas do vírus da influenza tipo B, como Engl/73, e SP/83, verificam-se médias de $5,13(\delta 1,45)$ e $4,89(\delta 1,26)$, respectivamente.

Analisando os resultados apresentados na Figura 7, pode-se verificar, no período da avaliação sorológica, que cepas mais recentemente incidentes na população de São Paulo, como os subtipos $\left(\mathrm{H}_{1} \mathrm{~N}_{1}\right)$ e $\left(\mathrm{H}_{3} \mathrm{~N}_{2}\right)$ do vírus influenza tipo $\mathrm{A}$, apresentam maiores médias de títulos de anticorpos comparados aos mesmos subtipos menos recentes. No que se refere ao tipo $B$ desse vírus, a situação é inversa, pois a cepa $\mathrm{B} / \mathrm{Engl} / 73$, apesar de registrar incidência mais antiga do que a cepa $\mathrm{B} / \mathrm{SP} / 83$, apresentou a mais alta média de títulos.

Devido a maior reatogenicidade de cepa B/Engl/ 73 , sobre as demais cepas dos vírus de influenza avaliadas e apoiados na doutrina do "Pecado original antigênico", supõe-se que esta cepa tenha sido responsável pela primo infecção na maioria dos indivíduos desta investigação.

Em estudo anterior com os subtipos $\left(\mathrm{H}_{3} \mathrm{~N}_{2}\right)$, $\left(\mathrm{H}_{1} \mathrm{~N}_{1}\right)$ do vírus da influenza tipo $\mathrm{A}$ e o tipo $\mathrm{B}$, verificou-se que indivíduos vacinados contra os três antígenos tiveram respostas imune humoral de até $100 \%$ para o tipo B, $93,75 \%$ e $73,68 \%$ para os subtipos $\mathrm{A}\left(\mathrm{H}_{3} \mathrm{~N}_{2}\right)$ e $\left(\mathrm{H}_{1} \mathrm{~N}_{1}\right)$, respectivamente, prevalecendo, também, a imunogenicidade do tipo $B^{15}$.

Diversos trabalhos desenvolvidos com vírus da influenza tipo B consideram que as características menos variáveis deste tipo com relação ao tipo $\mathrm{A}$, o torna menos epidêmico. Assim, é mais freqüuente reincidir na população, cepas homólogas do que as heterólogas e para as quais o sistema imune do organismo infectado responde em altos níveis de anticorpos, como ocorre na reação específica de antígeno e anticorpo ${ }^{4,16,22,24}$.

Mancini e col. ${ }^{14}$, estudaram grupos de adultos e crianças vacinados contra influenza e verificaram maior índice de respostas imunes tanto do tipo específica como cruzada para adultos e quase totalmente do tipo específica para crianças, atribuindo a maior reatividade dos adultos o fato deles terem sido mais expostos à infecção natural da influenza.

Zuckerman e col. ${ }^{30}(1991)$, avaliaram através de um estudo realizado com cem estudantes voluntários, da Escola de Medicina de Londres, a resposta sorológica frente a vacinação. Estes autores, tam- bém, trabalharam com diferentes cepas, tipo $\mathrm{A}\left(\mathrm{H}_{1} \mathrm{~N}_{1}\right)$ e $\left(\mathrm{H}_{3} \mathrm{~N}_{2}\right)$, do vírus influenza, assim como o tipo B, concluindo que mais de $98 \%$ dos voluntários deveriam estar protegidos contra as cepas epidêmicas circulantes, dos típos $\mathrm{A}$ e $\mathrm{B}$ do vírus influenza.

Da avaliação sorológica realizada no presente trabalho, conclui-se que ambos tipos A e B do vírus da influenza apresentam acentuada imunogenicidade, que ficou demonstrada na amplitude das médias de níveis de anticorpos detectados na população estudada, o que indica considerável prevalência sorológica do vírus da influenza, na cidade de São Paulo.

Com base no objetivo desta investigação, a avaliação da capacidade imunogênica das referidas cepas do vírus da influenza caracterizou a importância da seleção de cepas recentemente incidentes na população, para constituição antigênica da vacina contra gripe, considerando, principalmente, as propriedades das cepas do tipo A.

\section{Agradecimentos}

À pesquisadora científica Elvira M.M. do Nascimento pela tabulação dos dados e ao professor Dr. Carlos Alberto Bragança, do Instituto de Matemática e Estatística da USP, pela análise estatística.

MANCINI, D.A.P. et al. [Serological surveillance of influenza A and B, at the universitarian students, Brazil]. Rev. Saúde públ., S. Paulo, 25: 468-72, 1991. Wide serological prevalence of influenza $A$ and $B$ was verified by the serological survey covering 200 students of the University of S. Paulo during the $1984-1985$ period. The humoral antibodies were detected by the single radial haemolysis technique, whose arithmetic titres averages were greater for both subtypes, $\left(\mathrm{H}_{1} \mathrm{~N}_{1}\right)$ and $\left(\mathrm{H}_{3} \mathrm{~N}_{2}\right)$ of the influenza $A$ virus strains recently isolated from the population. However, the situation of this type B virus was not the same as that of type A seeing that the B/Engl/ $847 / 73$, although an older strain, showed better reactogenicity than the other strains evaluated. It is possible that is was responsible for the primo infection of most of the components of the group surveyed, as the phenomenon of the "Original Antigenic Sin" explains. The antibody responses to subtypes of influenza $A$ and $B$ in this survey demonstrated levels with low SRH titres (2.5 to $3.5 \mathrm{~mm})$ and high SRH titres $(\geq 4.0 \mathrm{~mm})$ related to the lowest and highest levels of suggest protection against infection. Individual immunity and persistence of antibody are related to frequency and recent occurrence of exposition to influenza. As concerns the formulation of influenza vaccine it was established that this preserves the antigen selection of the new strains isolated from the population, mainly due to the behavior of the influenza $A$ virus.

Keywords: Influenza, epidemiology. Seroepidemiologic methods. 


\section{Referências Bibliográficas}

1. ALLING, D.W.; BLACKWELDER, W.C.; STUARTHARRIS, C.H. A excess mortality influenza epidemics in the United States, 1968-1976. Amer. J. Epidem., 113: 3041, 1981 .

2. ANGELOVA, L.A. Long term immunity to influenza $\left(\mathrm{H}_{l} \mathrm{~N}_{\mathrm{l}}\right)$ in humans. Ann. Virol, Paris, 113: 267-72, 1982.

3. BARKER, W.H. \& MULLOLY, J.P. Impact of epidemic type A influenza in a define adult population. Amer. J. Epidem., 112: $798-811,1980$

4. CATE, T.R. Clinical manifestation and consequences of influenza. Amer. J. Med., 82(6A): 15-8, 1987.

5. CENTER FOR DISEASE CONTROL. Influenza vaccine efficiency in nursing home: outbreak reported during 198182. Morb. Mort. wkly Rep., 31: 190-7, 1982.

6. CHAVES, J.R.S.; NASCIMENTO, J.P. do; PEREIRA, M.S. Influenza surveillance in Rio de Janeiro. A serological study. Rev. Microbiol., Rio de Janeiro, 13: 161-5, 1982.

7. CHAKRAVERTY, P. Comparison of haemagglutinationinhibition and single-radial-haemolysis techniques for detection of antibodies to influenza B virus. Arch. Virol. Vienna, 63: 285-9. 1980.

8. FASEKAS DE ST. GROTH, S.; WEBSTER, R.G. Disquisitions on original antigenic sin. 1 - Evidence in man. J. exp. Med., 124: 331-45, 1966.

9. FOY, H.M.; HALL, C; COONEY, M.K.; ALLAN, I.; FOX. J.P. Influenza surveillance by age target group. Amer. $J$. Epidem., 109: 582-7, 1975.

10. INFLUENZA in the World. Wkly epidemiol. Rec., 54: 25-8, 1979.

11. JACKSON, G.G. \& MULLDOON, R.L. Viruses causing common respiratory infections in man: studies in infections diseases research. Chicago, University of Chicago Press, 1975. p. 199-248: Influenza A (Asian).

12. KIM, H.W.; BRANDT, C.D.; ARROBIO, J.O.; MURPHY, D; CHANOCKY, R.M.; PARROTT, R.H. Influenza A and $B$ virus infection in infants and young children the year - 1957-1976. Amer. J. Epidem., 109: 469-79, 1979.

13. LAYDE, P.M.; ENGELBERG, A.L.; DOBBS, H.I.; CURTIS, A.C.; CRAVEN, R.B.; GRAITCER, P.L.; SEDMAK, G.V.; ERICKON, J.D.; NOBLE, G.R. Outbreak of influenza A/ URSS/77 at Marquette University. J. infect. Dis., 142: 347$52,1980$.

14. MANCINI, G.; DONATELLI, G.; RUIZ, R.; ROZERA, C. Detection of antibodies and antigenic determinations on the haemagglutinin of influenza viruses A/Texas/1/77 and A/Bang Kok/1/79 in human sera. Arch. Virol, Vienna, 79: $117-21,1984$

15. MANCINI, D.A.P.; NASCIMENTO, E.M.M; YANO, A.F.B.; TAVARES, V.R. Avaliação da imunogenicidade das cepas $A\left(\mathrm{H}_{1} \mathrm{~N}_{1}\right)$ e $\mathrm{B}$, do vírus influenza, componentes da vacina contra gripe produzida pelo Instituto Butantan. Rev. Farm. Bioq. Univ. S. Paulo, 23(1): 1-10, 1987.

16. MTTCHELL, D.M.; MCMICHAEL, A.J.; LAMB, J.R. The immunology of influenza. Brit. med. Bull., 41: 80-5, 1985.

17. NASCIMENTO, J.P.; CHAVES, J.R.S.; PEREIRA, M.S. Single Radial Haemolysis: a sensitive test for the detection of antibody to influenza B virus. Rev. Microbiol., S. Paulo, 13(11): 65-9, 1982

18. ORGANIZATION MONDIALE DE LA SANTÉ. Comité d'Experts de la Standardisation Biologique, Genève, 1978. 30'me rapport. Genève, 1979. Annexe 3, p. 161-78: Normes revisees au vaccin antigripal (inactive). (Serie de Rapports Techniques, 638).

19. OXFORD, J.S.; YETTS, R.; SCHID, G.S. Quantification and analysis of the specificity of post-immunization antibodies to influenza B viruses using Single-Radial-Haemolysis. $J$ Hyg., 88: 325-33, 1982.

20. PONS, V.G; CANTER, J.; DOLIN, R. Influenza A/URSS/77 $\left(\mathrm{H}_{3} \mathrm{~N}_{2}\right)$ on a University Campus. Amer. J. Epidem., 111: 23-30, 1980

21. RUSSEL, S.M.; MCCAHON, D.; BEARE, A.S. Single - Radial - Haemolysis technique for the measurement of influenza antibody. J. gen. Virol. London, 27: 1-10, 1975.

22. SCHILD, G.C.; PEREIRA, M.S.; CHAKRAVERTY, P.; COLEMAN, M.T.; DOWDLE, M.W.; CHANG, M.K. Antigenic variants of influenza B virus. Brit. med. J., 4: 127-31, 1973.

23. SCHILD, F.C.; PEREIRA, M.S.; CHAKRAVERTY, P.; Single - Radial - Haemolysis: a new methods for the assay of antibody to influenza haemagglutinin. Bull. Wld Hlth Org. 53: $43-50,1975$.

24. SCHULMAN, J.L. Immunology of influenza. In: Kilborne, E.D., ed. The influenza viruses and influenza. New York, Academic Press, 1975. p. 373-93.

25. TAKIMOTO, S.; PANNUTI, C.S.; SALLES GOMES, L.F.; BARBOSA, H.G.; MORAES, V.C.M.; HIGUCHI, A.E.C Influenza em São Paulo durante os anos 1976-1978. Rev. Inst. Med. Trop. S. Paulo, 24(1): 49-55, 1982.

26. VELAZCO, J.G.; COUGH, R.B.; SIX, H.R.; GLEZE, W.P Surveillance of influenza in Houston from A/Victoria/73 $\left(\mathrm{H}_{3} \mathrm{~N}_{2}\right)$ to $\mathrm{A} / \mathrm{Texas} / 77\left(\mathrm{H}_{3} \mathrm{~N}_{2}\right)$. Predominance and antigenic characterization of "Intermediate" Strains. Bull. Wld Hlth Org., 61: 345-52, 1983.

27. WORLD HEALTH ORGANTZATION. Recommended composition of influenza virus vaccine for use in the 1985-86 season. Wkly epidemiol. Rec., 60: 53-60, 1985.

28. YAMAGISH, H,; NAGASMINE, T.; SHIMODA, K.; SEYA I,; IGARASHI, Y.; YOSHIDA, I.; MATMOTO, M Comparative measurements of equine influenza virus antibodies in horse sera by SHR, neutral and HI tests. $J$. clin. Microb., 15: 660-2, 1982.

29. ZUCKERMAN, M.; OXFORD, Y.; WOOD, J.; TAYLOR, J. Influenza $A\left(\mathrm{H}_{3} \mathrm{~N}_{2}\right)$ component of recommended vaccine induces antibody to current virus. Lancet, 335: 179-80, 1990.

30. ZUCKERMANN, M.; WOOD, J.; CHAKRAVERTY, P.; TAYLOR, J.; HEALTH, A.; OXFORD, J. Serological responses in volunteers to inactivated trivalent subunit influenza vaccine: antibody reactivity with epidemic influenza $A$ and $B$ strains and evidence of a rapid immune response. J. med. Virol., 33: 133-7, 1991.

Recebido para publicaçāo em 02/05/1991. Reapresentado em 26/08/1991. Aprovado para publicação em 30/08/1991. 